\title{
On the Expected Discounted Penalty Function at Ruin of a Surplus Process with Interest
}

\author{
by \\ Jun Cai and David C.M. Dickson \\ The University of Melbourne \\ RESEARCH PAPER NUMBER 91
}

November 2001

Centre for Actuarial Studies

Department of Economics

The University of Melbourne

Victoria 3010

Australia 


\title{
On the expected discounted penalty function at ruin of a surplus process with interest
}

\author{
Jun Cai and David C. M. Dickson \\ Centre for Actuarial Studies \\ Faculty of Economics and Commerce \\ University of Melbourne \\ Victoria 3010 \\ Australia
}

\begin{abstract}
In this paper, we study the expected value of a discounted penalty function at ruin of the classical surplus process modified by the inclusion of interest on the surplus. The 'penalty' is simply a function of the surplus immediately prior to ruin and the deficit at ruin. An integral equation for the expected value is derived, while the exact solution is given when the initial surplus is zero. Dickson's (1992) formulae for the distribution of the surplus immediately prior to ruin in the classical surplus process are generalised to our modified surplus process.
\end{abstract}

Keywords: ruin penalty function, surplus prior to ruin, deficit at ruin, Laplace transform, Volterra equation, compound Poisson process, force of interest.

\section{Introduction}

Consider a compound Poisson risk model. Assume that $T_{n}=\sum_{k=1}^{n} Y_{k}$ is the time of the $n$-th claim and $X_{n}$ is the amount of the $n$-th claim. Suppose that $\left\{X_{n}, n \geq 1\right\}$ and $\left\{Y_{n}, n \geq 1\right\}$ are two independent sequences of i.i.d. positive random variables, where $\left\{X_{n}, n \geq 1\right\}$ have common distribution $F(x)=\operatorname{Pr}\left\{X_{1} \leq x\right\}$ with mean $\mu>0$, and $\left\{Y_{n}, n \geq 1\right\}$ have common exponential distribution $\operatorname{Pr}\left\{Y_{1} \leq x\right\}=1-\exp \{-\lambda x\}, x \geq 0$, where $\lambda>0$.

The number of claims up to time $t$ is denoted by $N(t)=\sup \left\{n: T_{n} \leq t\right\}$. The claim number process is a Poisson process with rate $\lambda$, and the aggregate claim amount up to time $t$ is

$$
Z(t)=\sum_{n=1}^{N(t)} X_{n}
$$


Assume that the insurer receives interest on its surplus at a constant force $\delta$ per unit time. Let $U_{\delta}(t)$ denote the surplus at time $t$. Then

$$
U_{\delta}(t)=u e^{\delta t}+c \bar{s}_{\bar{t} \mid}^{(\delta)}-\int_{0}^{t} e^{\delta(t-x)} d Z(x)
$$

where $u$ is the initial surplus and $c=(1+\theta) \lambda \mu$ is the rate of premium income per unit time, where $\theta>0$ is the premium loading factor.

Let the time of ruin be

$$
T_{\delta}=\left\{\begin{array}{l}
\inf \left\{t: U_{\delta}(t)<0\right\} \\
\infty \text { if } U_{\delta}(t) \geq 0 \text { for all } t>0 .
\end{array}\right.
$$

Denote by $\psi_{\delta}(u)$ the ruin probability for the surplus process given by equation (1.1). Then

$$
\psi_{\delta}(u)=\operatorname{Pr}\left\{T_{\delta}<\infty\right\}=\operatorname{Pr}\left\{\cup_{t \geq 0}\left(U_{\delta}(t)<0\right)\right\}
$$

The following notation applies throughout this paper:

$$
\begin{aligned}
f(x) & =\frac{d}{d x} F(x) \\
F_{1}(x) & =1-\bar{F}_{1}(x)=\frac{1}{\mu} \int_{0}^{x} \bar{F}(t) d t \\
\bar{\psi}_{\delta}(u) & =1-\psi_{\delta}(u) ; \psi(u)=\psi_{\delta=0}(u) ; \quad \psi(0)=\frac{\lambda \mu}{c}=\frac{1}{1+\theta} ; \\
U\left(T_{\delta}^{-}\right) & =\text {the surplus immediately prior to ruin; } \\
\left|U\left(T_{\delta}\right)\right| & =\text { the deficit at ruin; } \\
F_{\delta}(u, x) & =\operatorname{Pr}\left\{U\left(T_{\delta}^{-}\right) \leq x, T_{\delta}<\infty\right\} ; f_{\delta}(u, x)=\frac{d}{d x} F_{\delta}(u, x) \\
H_{\delta}(u, x, y) & =\operatorname{Pr}\left\{U\left(T_{\delta}^{-}\right) \leq x,\left|U\left(T_{\delta}\right)\right| \leq y, T_{\delta}<\infty\right\} \\
h_{\delta}(u, x, y) & =\frac{\partial^{2}}{\partial x \partial y} H_{\delta}(u, x, y) .
\end{aligned}
$$

We consider the expected value of a discounted function of the surplus immediately prior to ruin and the deficit at ruin when ruin occurs as a function of the initial surplus $u$, namely,

$$
\Phi_{\delta, \alpha}(u)=E\left(w\left(U\left(T_{\delta}^{-}\right),\left|U\left(T_{\delta}\right)\right|\right) e^{-\alpha T_{\delta}} I\left(T_{\delta}<\infty\right)\right)
$$

where $I(A)$ is the indicator function of a set $A, w$ is a non-negative function, and $\alpha$ is a non-negative valued parameter. We can interpret $\exp \left\{-\alpha T_{\delta}\right\}$ as the 'discounting factor'.

The function $\Phi_{\delta, \alpha}(u)$ provides a unified means of studying the joint distribution of the surplus immediately prior to ruin and the deficit at ruin. The distributions of these quantities, both joint and marginal, have been studied by many authors including Dickson (1992), 
Dufresne and Gerber (1988), Gerber et al (1987), Gerber and Shiu (1997, 1998) and Lin and Willmot (1999). In particular, Gerber and Shiu (1998) studied the function $\Phi_{\delta=0, \alpha}(u)$ in detail, but they did not consider the case when $\delta>0$.

In this paper, we will follow ideas in Sundt and Teugels (1995). In particular, we will consider the function $\Phi_{\delta, \alpha=0}(u)=\Phi_{\delta}(u)$. We will also derive an integral equation for $\Phi_{\delta, \alpha}(u)$ and find the Laplace transform of an auxiliary function of $\Phi_{\delta, \alpha}(u)$. We then find an exact solution for $\Phi_{\delta}(0)$ and generalise Dickson's (1992) formulae for the distribution of the surplus prior to ruin when $\delta=0$ to the situation when $\delta>0$. Applications of the results will be illustrated by a variety of examples.

\section{Integral equations}

Using similar arguments to Gerber and Shiu (1998) and Sundt and Teugels (1995), we condition on the time, $t$, and on the amount, $x$, of the first claim. We note that if $x \leq$ $u e^{\delta t}+c \bar{s}_{\bar{t} \mid}^{(\delta)}$, then ruin does not occur, but if $x>u e^{\delta t}+c \bar{s}_{\bar{t} \mid}^{(\delta)}$, then ruin occurs. Thus,

$$
\begin{aligned}
\Phi_{\delta, \alpha}(u)= & \int_{0}^{\infty} \lambda e^{-\lambda t} \int_{0}^{\infty} E\left(w\left(U\left(T_{\delta}^{-}\right),\left|U\left(T_{\delta}\right)\right|\right) e^{-\alpha T_{\delta}} I\left(T_{\delta}<\infty\right) \mid X_{1}=x, Y_{1}=t\right) d F(x) d t \\
= & \int_{0}^{\infty} \lambda e^{-(\lambda+\alpha) t} \int_{0}^{u e^{\delta t}+c \bar{s}_{\bar{t} \mid}^{(\delta)}} \Phi_{\delta, \alpha}\left(u e^{\delta t}+c \bar{s}_{\bar{t} \mid}^{(\delta)}-x\right) d F(x) d t \\
& +\int_{0}^{\infty} \lambda e^{-(\lambda+\alpha) t} \int_{u e^{\delta t}+c \bar{s}_{\bar{t} \mid}^{(\delta)}}^{\infty} w\left(u e^{\delta t}+c \bar{s}_{\bar{t} \mid}^{(\delta)}, x-u e^{\delta t}-c \bar{s}_{\bar{t} \mid}^{(\delta)}\right) d F(x) d t .
\end{aligned}
$$

Substituting $y=u e^{\delta t}+c \bar{s}_{\bar{t} \mid}^{(\delta)}=u e^{\delta t}+c\left(e^{\delta t}-1\right) / \delta$ in the above equation, we have

$$
\begin{aligned}
\Phi_{\delta, \alpha}(u)= & \lambda(\delta u+c)^{\frac{\lambda+\alpha}{\delta}} \int_{u}^{\infty}(\delta y+c)^{-\frac{\lambda+\alpha}{\delta}-1} \int_{0}^{y} \Phi_{\delta, \alpha}(y-x) d F(x) d y \\
& +\lambda(\delta u+c)^{\frac{\lambda+\alpha}{\delta}} \int_{u}^{\infty}(\delta y+c)^{-\frac{\lambda+\alpha}{\delta}-1} \int_{y}^{\infty} w(y, x-y) d F(x) d y \\
= & \lambda(\delta u+c)^{\frac{\lambda+\alpha}{\delta}} \int_{u}^{\infty}(\delta y+c)^{-\frac{\lambda+\alpha}{\delta}-1}\left(\int_{0}^{y} \Phi_{\delta, \alpha}(y-x) d F(x)+A(y)\right) d y
\end{aligned}
$$

where

$$
A(t)=\int_{t}^{\infty} w(t, s-t) d F(s)
$$

Differentiating equation (2.1) with respect to $u$, we get

$$
\frac{d}{d u} \Phi_{\delta, \alpha}(u)=\frac{\lambda+\alpha}{c+\delta u} \Phi_{\delta, \alpha}(u)-\frac{\lambda}{c+\delta u}\left(\int_{0}^{u} \Phi_{\delta, \alpha}(u-x) d F(x)+A(u)\right) .
$$


Replacing $u$ by $t$ in equation (2.2) and re-arranging, we get for any $t \geq 0$,

$$
(\lambda+\alpha) \Phi_{\delta, \alpha}(t)=(c+\delta t) \frac{d}{d t} \Phi_{\delta, \alpha}(t)+\lambda \int_{0}^{t} \Phi_{\delta, \alpha}(t-s) d F(s)+\lambda A(t) .
$$

Thus, integrating equation (2.3) from 0 to $u$, then performing integration by parts, we get,

$$
\begin{aligned}
& (\lambda+\alpha) \int_{0}^{u} \Phi_{\delta, \alpha}(t) d t \\
= & \int_{0}^{u}(c+\delta t) d \Phi_{\delta, \alpha}(t)+\lambda \int_{0}^{u} \int_{0}^{t} \Phi_{\delta, \alpha}(t-s) d F(s) d t+\lambda \int_{0}^{u} A(t) d t \\
= & (c+\delta u) \Phi_{\delta, \alpha}(u)-c \Phi_{\delta, \alpha}(0)-\delta \int_{0}^{u} \Phi_{\delta, \alpha}(t) d t+\lambda \int_{0}^{u} \int_{s}^{u} \Phi_{\delta, \alpha}(t-s) d t d F(s)+\lambda \int_{0}^{u} A(t) d t \\
= & (c+\delta u) \Phi_{\delta, \alpha}(u)-c \Phi_{\delta, \alpha}(0)-\delta \int_{0}^{u} \Phi_{\delta, \alpha}(t) d t+\lambda \int_{0}^{u}\left(\int_{0}^{u-s} \Phi_{\delta, \alpha}(y) d y\right) d F(s)+\lambda \int_{0}^{u} A(t) d t \\
= & (c+\delta u) \Phi_{\delta, \alpha}(u)-c \Phi_{\delta, \alpha}(0)-\delta \int_{0}^{u} \Phi_{\delta, \alpha}(t) d t+\lambda \int_{0}^{u} F(s) \Phi_{\delta, \alpha}(u-s) d s+\lambda \int_{0}^{u} A(t) d t,
\end{aligned}
$$

which implies that

$$
(c+\delta u) \Phi_{\delta, \alpha}(u)=c \Phi_{\delta, \alpha}(0)-\lambda \int_{0}^{u} A(t) d t+\int_{0}^{u}(\delta+\alpha+\lambda \bar{F}(u-t)) \Phi_{\delta, \alpha}(t) d t
$$

or

$$
\begin{aligned}
\Phi_{\delta, \alpha}(u) & =\frac{c \Phi_{\delta, \alpha}(0)}{c+\delta u}-\frac{\lambda}{c+\delta u} \int_{0}^{u} A(t) d t+\int_{0}^{u} \frac{\delta+\alpha+\lambda \bar{F}(u-t)}{c+\delta u} \Phi_{\delta, \alpha}(t) d t \\
& =\frac{c \Phi_{\delta, \alpha}(0)}{c+\delta u}-\frac{\lambda}{c+\delta u} \int_{0}^{u} A(t) d t+\int_{0}^{u} k_{\delta, \alpha}(u, t) \Phi_{\delta, \alpha}(t) d t
\end{aligned}
$$

where

$$
k_{\delta, \alpha}(u, t)=\frac{\delta+\alpha+\lambda \bar{F}(u-t)}{c+\delta u} .
$$

In particular, recalling that $\Phi_{\delta}(u)=\Phi_{\delta, \alpha=0}(u)$, we get

$$
(c+\delta u) \Phi_{\delta}(u)=c \Phi_{\delta}(0)-\lambda \int_{0}^{u} A(t) d t+\int_{0}^{u}(\delta+\lambda \bar{F}(u-t)) \Phi_{\delta}(t) d t
$$

for any $u \geq 0$, and

$$
\Phi_{\delta}(u)=\frac{c \Phi_{\delta}(0)}{c+\delta u}-\frac{\lambda}{c+\delta u} \int_{0}^{u} A(t) d t+\int_{0}^{u} k_{\delta}(u, t) \Phi_{\delta}(t) d t,
$$

where

$$
k_{\delta}(u, t)=k_{\delta, \alpha=0}(u, t)=\frac{\delta+\lambda \bar{F}(u-t)}{c+\delta u}
$$


Both equations (2.4) and (2.6) are types of the following Volterra integral equation

$$
\varphi(x)=l(x)+\int_{0}^{x} k(x, s) \varphi(s) d s
$$

It is well known (see, for example, Mikhlin(1957)) that if $l$ is absolutely integrable and the kernel $k$ is continuous, then for any $x>0$, the unique solution for $\varphi(x)$ has the following representation

$$
\varphi(x)=l(x)+\int_{0}^{x} K(x, s) l(s) d s
$$

where

$$
K(x, s)=\sum_{m=1}^{\infty} k_{m}(x, s), \quad x>s \geq 0,
$$

is called the resolvent of equation (2.7), and

$$
k_{m}(x, s)=\int_{s}^{x} k(x, t) k_{m-1}(t, s) d t, \quad m=2,3, \ldots, \quad x>s \geq 0,
$$

with $k_{1}(x, s)=k(x, s)$.

Further, $\varphi(x)$ can be approximated recursively by Picard's sequence (see Mikhlin(1957)) defined by

$$
\varphi_{n}(x)=l(x)+\int_{0}^{x} \varphi_{n-1}(s) l(s) d s, n=1,2, \ldots
$$

with $\varphi_{0}(x)=l(x)$

Therefore, at least in principle, if we can find $\Phi_{\delta, \alpha}(0)$, we can find the form of the solution for $\Phi_{\delta, \alpha}(u)$ and can approximate $\Phi_{\delta, \alpha}(u)$ recursively. Hence it is important to be able to find $\Phi_{\delta, \alpha}(0)$. Gerber and Shiu (1998) have obtained $\Phi_{\delta=0, \alpha}(0)$ using the technique of probability measure transform. However, we will find $\Phi_{\delta}(0)=\Phi_{\delta, \alpha=0}(0)$ by using Laplace transforms in the next section. In what follows, unless we state otherwise, the term Laplace transform refers to a Stieltjes transform.

\section{The exact solution for $\Phi_{\delta}(0)$}

We define an auxiliary function of $\Phi_{\delta}(u)$ as

$$
Z_{\delta}(u)=\frac{\Phi_{\delta}(0)-\Phi_{\delta}(u)}{\Phi_{\delta}(0)}
$$


Then $Z_{\delta}(0)=0$. Also, if the claim size distribution $F$ is sufficiently regular, then $\Phi_{\delta}(u) \rightarrow 0$ as $u \rightarrow \infty$. In this case, $\lim _{u \rightarrow \infty} Z_{\delta}(u)=1$ and we can find the Laplace transform of $Z_{\delta}$, namely,

$$
\gamma_{\delta}(s)=\int_{0}^{\infty} e^{-s x} d Z_{\delta}(x)
$$

with $\gamma_{\delta}(0)=1$. Therefore, we assume that $\Phi_{\delta}(u) \rightarrow 0$ as $u \rightarrow \infty$. In particular, a sufficient condition for this assumption is that $w$ is bounded. In fact, if $w \leq L$ for some $L>0$ and $F$ has a finite second moment, then

$$
\begin{aligned}
(c+\delta u) \Phi_{\delta}(u) & \leq L(c+\delta u) E I\left(T_{\delta}<\infty\right) \\
& =L\left(c \psi_{\delta}(u)+\delta u \psi_{\delta}(u)\right) \leq L(c \psi(u)+\delta u \psi(u)) \rightarrow 0
\end{aligned}
$$

as $u \rightarrow \infty$. Thus, letting $u \rightarrow \infty$ in equation (2.5), we get

$$
\Phi_{\delta}(0)=\frac{\lambda}{c} \int_{0}^{\infty} A(t) d t-\frac{\delta}{c} \int_{0}^{\infty} \Phi_{\delta}(t) d t
$$

Hence, $\Phi_{\delta}(0)$ can also be obtained by finding the standard Laplace transform of $\Phi_{\delta}$, namely $\int_{0}^{\infty} e^{-s t} \Phi_{\delta}(t) d t$

Now, equation (3.1) implies that

$$
\Phi_{\delta}(u)=\Phi_{\delta}(0)-\Phi_{\delta}(0) Z_{\delta}(u)
$$

Inserting equation (3.3) into equation (2.5), we get

$$
\begin{aligned}
& c \Phi_{\delta}(0)-c \Phi_{\delta}(0) Z_{\delta}(u)+\delta \Phi_{\delta}(0) u-\delta \Phi_{\delta}(0) u Z(u) \\
= & c \Phi_{\delta}(0)-\lambda \int_{0}^{u} A(t) d t+\delta \int_{0}^{u}\left(\Phi_{\delta}(0)-\Phi_{\delta}(0) Z_{\delta}(t)\right) d t \\
& +\lambda \int_{0}^{u} \bar{F}(u-t)\left(\Phi_{\delta}(0)-\Phi_{\delta}(0) Z_{\delta}(t)\right) d t
\end{aligned}
$$

which implies that

$$
(c+\delta u) Z_{\delta}(u)=\delta \int_{0}^{u} Z_{\delta}(t) d t+\frac{\lambda m_{A}}{\Phi_{\delta}(0)} A_{1}(u)-\lambda \mu F_{1}(u)+\lambda \mu Z_{\delta} * F_{1}(u)
$$

where

$$
A_{1}(u)=\frac{1}{m_{A}} \int_{0}^{u} A(t) d t, \quad m_{A}=\int_{0}^{\infty} A(t) d t,
$$

and $Z_{\delta} * F_{1}$ is the Stieltjes convolution of $Z_{\delta}$ and $F_{1}$. 
Thus, differentiating both sides of equation (3.4), we have

$$
(c+\delta u) d Z_{\delta}(u)+\delta Z_{\delta}(u) d u=\delta Z_{\delta}(u) d u+\frac{\lambda m_{A}}{\Phi_{\delta}(0)} d A_{1}(u)-\lambda \mu d F_{1}(u)+\lambda \mu d Z_{\delta} * F_{1}(u)
$$

which gives

$$
(c+\delta u) d Z_{\delta}(u)=\frac{\lambda m_{A}}{\Phi_{\delta}(0)} d A_{1}(u)-\lambda \mu d F_{1}(u)+\lambda \mu d Z_{\delta} * F_{1}(u)
$$

Taking Laplace transforms of both sides of equation (3.5) yields

$$
c \gamma_{\delta}(s)-\delta \frac{d}{d s} \gamma_{\delta}(s)=\frac{\lambda m_{A}}{\Phi_{\delta}(0)} \beta(s)-\lambda \mu \phi(s)+\lambda \mu \phi(s) \gamma_{\delta}(s),
$$

where $\phi$ and $\beta$ are the Laplace transforms of $F_{1}$ and $A_{1}$ respectively, namely,

$$
\phi(s)=\int_{0}^{\infty} e^{-s x} d F_{1}(x)=\frac{1}{\mu} \int_{0}^{\infty} e^{-s x} \bar{F}(x) d x
$$

or, if $F$ is a continuous distribution,

$$
\int_{0}^{\infty} e^{-s x} f(x) d x=1-\mu s \phi(s),
$$

and

$$
\beta(s)=\int_{0}^{\infty} e^{-s x} d A_{1}(x)=\frac{1}{m_{A}} \int_{0}^{\infty} e^{-s x} A(x) d x
$$

Equation (3.6) is equivalent to

$$
-\delta \frac{d}{d s} \gamma_{\delta}(s)+P_{\delta}(s) \gamma_{\delta}(s)=Q_{\delta}(s)
$$

where

$$
P_{\delta}(s)=c-\lambda \mu \phi(s)
$$

and

$$
Q_{\delta}(s)=\frac{\lambda m_{A}}{\Phi_{\delta}(0)} \beta(s)-\lambda \mu \phi(s)
$$

When $\delta>0$, we note that

$$
\frac{d}{d s}\left(\gamma_{\delta}(s) \exp \left(-\frac{1}{\delta} \int_{0}^{s} P_{\delta}(t) d t\right)\right)=-\frac{1}{\delta} Q_{\delta}(s) \exp \left(-\frac{1}{\delta} \int_{0}^{s} P_{\delta}(t) d t\right)
$$


and using the arguments of Sundt and Teugels (1995), we get

$$
\gamma_{\delta}(s) \exp \left(-\frac{1}{\delta} \int_{0}^{s} P_{\delta}(t) d t\right)=\frac{1}{\delta} \int_{s}^{\infty} Q_{\delta}(t) \exp \left(-\frac{1}{\delta} \int_{0}^{t} P_{\delta}(z) d z\right) d t
$$

Hence, $\gamma_{\delta}(0)=1$ gives

$$
\begin{aligned}
\delta & =\int_{0}^{\infty} Q_{\delta}(t) \exp \left(-\frac{1}{\delta} \int_{0}^{t} P_{\delta}(z) d z\right) d t \\
& =\frac{\lambda m_{A}}{\Phi_{\delta}(0)} \int_{0}^{\infty} \beta(t) \exp \left(-\frac{1}{\delta} \int_{0}^{t} P_{\delta}(z) d z\right) d t-\lambda \mu \int_{0}^{\infty} \phi(t) \exp \left(-\frac{1}{\delta} \int_{0}^{t} P_{\delta}(z) d z\right) d t
\end{aligned}
$$

or, equivalently,

$$
\begin{aligned}
\Phi_{\delta}(0) & =\frac{\lambda m_{A} \int_{0}^{\infty} \beta(t) \exp \left(-\frac{1}{\delta}\left(c t-\lambda \mu \int_{0}^{t} \phi(s) d s\right)\right) d t}{\delta+\lambda \mu \int_{0}^{\infty} \phi(t) \exp \left(-\frac{1}{\delta}\left(c t-\lambda \mu \int_{0}^{t} \phi(s) d s\right)\right) d t} \\
& =\frac{\lambda m_{A}}{\kappa_{\delta}} \int_{0}^{\infty} \beta(\delta z) \exp \left(-c z+\lambda \mu \int_{0}^{z} \phi(\delta s) d s\right) d z
\end{aligned}
$$

where equation (3.9) follows from the substitution $t=\delta z$, and we define

$$
\begin{aligned}
\kappa_{\delta} & =1+\lambda \mu \int_{0}^{\infty} \phi(\delta z) \exp \left(-c z+\lambda \mu \int_{0}^{z} \phi(\delta s) d s\right) d z \\
& =c \int_{0}^{\infty} \exp \left(-c z+\lambda \mu \int_{0}^{z} \phi(\delta s) d s\right) d z
\end{aligned}
$$

using integration by parts.

Note that $\kappa_{\delta}$ does not depend on the choice of $A$ or $w$, but $\beta$ does. We will illustrate the applications of equations (2.6) and (3.9) by examples.

Similarly, if we define an auxiliary function of $\Phi_{\delta, \alpha}(u)$ as

$$
Z_{\delta, \alpha}(u)=\frac{\Phi_{\delta, \alpha}(0)-\Phi_{\delta, \alpha}(u)}{\Phi_{\delta, \alpha}(0)}
$$

and denote the Laplace transform of $Z_{\delta, \alpha}$ by

$$
\gamma_{\delta, \alpha}(s)=\int_{0}^{\infty} e^{-s x} d Z_{\delta, \alpha}(x)
$$

we get a differential equation for $\gamma_{\delta, \alpha}(s)$, namely

$$
c \gamma_{\delta, \alpha}(s)-\delta \frac{d}{d s} \gamma_{\delta, \alpha}(s)=\alpha \frac{\gamma_{\delta, \alpha}(s)}{s}+\frac{\lambda m_{A}}{\Phi_{\delta}(0)} \beta(s)-\frac{\alpha}{s}-\lambda \mu \phi(s)+\lambda \mu \phi(s) \gamma_{\delta, \alpha}(s)
$$

Unfortunately, we are unable to determine $\gamma_{\delta, \alpha}(0)$ using the methods of this section. It seems that the method of Gerber and Shiu (1998) does not apply either. 
Example 3.1 Let $w\left(x_{1}, x_{2}\right)=1$. Then $\Phi_{\delta}(u)=\psi_{\delta}(u), A(t)=\bar{F}(t), m_{A}=\mu$, and $\beta(s)=$ $\phi(s)$, and equation (2.6) gives

$$
\psi_{\delta}(u)=\frac{c \psi_{\delta}(0)}{c+\delta u}-\frac{\lambda \mu}{c+\delta u} F_{1}(u)+\int_{0}^{u} k_{\delta}(u, t) \psi_{\delta}(t) d t
$$

Equivalently, as $\bar{\psi}_{\delta}(u)=1-\psi_{\delta}(u)$,

$$
\bar{\psi}_{\delta}(u)=\frac{c \bar{\psi}_{\delta}(0)}{c+\delta u}+\int_{0}^{u} k_{\delta}(u, t) \bar{\psi}_{\delta}(t) d t
$$

which is equation (2) of Sundt and Teugels (1995). In addition, equations (3.9) and (3.10) give

$$
\psi_{\delta}(0)=\frac{\lambda \mu}{\kappa_{\delta}} \int_{0}^{\infty} \phi(\delta z) \exp \left(-c z+\lambda \mu \int_{0}^{z} \phi(\delta s) d s\right) d z=\frac{\kappa_{\delta}-1}{\kappa_{\delta}}
$$

which is equivalent to equation (14) of Sundt and Teugels (1995). As $1 / \kappa_{\delta}=\bar{\psi}_{\delta}(0)$, the general expression for $\Phi_{\delta}(0)$ in (3.9) becomes

$$
\Phi_{\delta}(0)=\lambda m_{A} \bar{\psi}_{\delta}(0) \int_{0}^{\infty} \beta(\delta z) \exp \left(-c z+\lambda \mu \int_{0}^{z} \phi(\delta s) d s\right) d z
$$

Example 3.2 Let $w\left(x_{1}, x_{2}\right)=I\left(x_{1} \leq x\right) I\left(x_{2} \leq y\right)$. Then $\Phi_{\delta}(u)=H_{\delta}(u, x, y)$ and

$$
\begin{aligned}
A(t) & =\int_{t}^{\infty} w(t, s-t) d F(s)=\int_{t}^{\infty} I(t \leq x) I(s-t \leq y) d F(s) \\
& =I(t \leq x) \int_{t}^{t+y} d F(s)=I(t \leq x)(\bar{F}(t)-\bar{F}(t+y)) .
\end{aligned}
$$

Thus, equation (2.6) gives

$$
\begin{aligned}
H_{\delta}(u, x, y)= & \frac{c H_{\delta}(0, x, y)}{c+\delta u}-\frac{\lambda}{c+\delta u} \int_{0}^{u \wedge x}(\bar{F}(t)-\bar{F}(y+t)) d t \\
& +\int_{0}^{u} k_{\delta}(u, t) H_{\delta}(t, x, y) d t \\
= & \frac{c H_{\delta}(0, x, y)}{c+\delta u}-\frac{\lambda \mu}{c+\delta u}\left[F_{1}(u \wedge x)+F_{1}(y)-F_{1}(u \wedge x+y)\right] \\
& +\int_{0}^{u} k_{\delta}(u, t) H_{\delta}(t, x, y) d t .
\end{aligned}
$$

In addition,

$$
\beta(t)=\frac{1}{m_{A}} \int_{0}^{\infty} e^{-t s} A(s) d s=\frac{1}{m_{A}} \int_{0}^{x} e^{-t s}(\bar{F}(s)-\bar{F}(y+s)) d s .
$$

Hence, equation (3.9) gives

$$
H_{\delta}(0, x, y)=\frac{\lambda}{\kappa_{\delta}} \int_{0}^{\infty} \int_{0}^{x} e^{-\delta s z}(\bar{F}(s)-\bar{F}(y+s)) d s \exp \left(-c z+\lambda \mu \int_{0}^{z} \phi(\delta s) d s\right) d z .
$$

Thus, equations (3.14) and (3.15) give the main results of Yang and Zhang (2001a). Similarly, letting $x \rightarrow \infty$ in these equations we get equations (4) and (12) of Yang and Zhang (2001b). 
Example 3.3 For $r \geq 0$, let $w\left(x_{1}, x_{2}\right)=e^{-r x_{2}}$. Then

$$
\Phi_{\delta}(u)=E\left(e^{-r\left|U\left(T_{\delta}\right)\right|} I\left(T_{\delta}<\infty\right)\right)=\tilde{W}_{\delta}(u, r),
$$

the Laplace transform of the deficit at ruin when ruin occurs. Thus, when $F$ is a continuous distribution,

$$
A(t)=\int_{t}^{\infty} w(t, x-t) d F(x)=\int_{t}^{\infty} e^{-r(x-t)} f(x) d x
$$

and by equations (3.8) and (3.7), we have

$$
\begin{aligned}
\beta(t) & =\frac{1}{m_{A}} \int_{0}^{\infty} e^{-t y} \int_{y}^{\infty} e^{-r(x-y)} f(x) d x d y \\
& =\frac{1}{m_{A}} \int_{0}^{\infty} e^{-r x} f(x) \int_{0}^{x} e^{-(t-r) y} d y d x=\frac{1}{m_{A}} \int_{0}^{\infty} f(x) \frac{e^{-r x}-e^{-t x}}{t-r} d x \\
& =\left(\frac{\mu}{m_{A}}\right) \frac{t \phi(t)-r \phi(r)}{t-r} .
\end{aligned}
$$

Thus, using equation (3.13) we get

$$
\tilde{W}_{\delta}(0, r)=\lambda \mu \bar{\psi}_{\delta}(0) \int_{0}^{\infty} \frac{\delta z \phi(\delta z)-r \phi(r)}{\delta z-r} \exp \left(-c z+\lambda \mu \int_{0}^{z} \phi(\delta s) d s\right) d z
$$

Therefore, given that $U_{\delta}(0)=0$ and ruin occurs, if the $(n+1)$-th moment of $F$ exists, we get the $n$-th moment of the deficit at ruin, namely,

$$
\begin{aligned}
& E\left(\left|U\left(T_{\delta}\right)\right|^{n} \mid T_{\delta}<\infty\right)=\frac{(-1)^{n}}{\psi_{\delta}(0)}\left(\left.\frac{d^{n}}{d r^{n}} \tilde{W}_{\delta}(0, r)\right|_{r=0}\right) \\
= & \frac{\lambda \mu \bar{\psi}_{\delta}(0)}{\psi_{\delta}(0)} \int_{0}^{\infty}\left(\left.\frac{d^{n}}{d r^{n}} \frac{\delta z \phi(\delta z)-r \phi(r)}{\delta z-r}\right|_{r=0}\right) \exp \left(-c z+\lambda \mu \int_{0}^{z} \phi(\delta s) d s\right) d z .
\end{aligned}
$$

In particular, when $n=1$ we have

$$
E\left(\left|U\left(T_{\delta}\right)\right| \mid T_{\delta}<\infty\right)=\frac{\lambda \mu \bar{\psi}_{\delta}(0)}{\psi_{\delta}(0)} \int_{0}^{\infty} \frac{1-\phi(\delta z)}{\delta z} \exp \left(-c z+\lambda \mu \int_{0}^{z} \phi(\delta s) d s\right) d z
$$

Similarly, we can let $w\left(x_{1}, x_{2}\right)=e^{-r\left(x_{1}+x_{2}\right)}$ to find the Laplace transform of $U\left(T^{-}\right)+|U(T)|$, the amount of the claim causing ruin when ruin occurs, and hence its moments.

\section{The distribution of the surplus prior to ruin}

Throughout this section we assume that $F$ is a continuous distribution with density $f$. From equation (3.15), we have

$$
h_{\delta}(0, x, y)=\frac{\partial^{2}}{\partial y \partial x} H_{\delta}(0, x, y)=\frac{\lambda}{\kappa_{\delta}} f(x+y) \int_{0}^{\infty} \exp \left\{-(c+\delta x) z+\lambda \mu \int_{0}^{z} \phi(\delta s) d s\right\} d z
$$


and

$$
F_{\delta}(0, x)=\lim _{y \rightarrow \infty} H_{\delta}(0, x, y)=\frac{\lambda}{\kappa_{\delta}} \int_{0}^{\infty} \int_{0}^{x} e^{-\delta s z} \bar{F}(s) d s \exp \left(-c z+\lambda \mu \int_{0}^{z} \phi(\delta s) d s\right) d z
$$

which gives

$$
f_{\delta}(0, x)=\frac{d}{d x} F_{\delta}(0, x)=\frac{\lambda}{\kappa_{\delta}} \bar{F}(x) \int_{0}^{\infty} \exp \left(-(c+\delta x) z+\lambda \mu \int_{0}^{z} \phi(\delta s) d s\right) d z
$$

Thus, equations (4.2) and (4.1) yield

$$
h_{\delta}(0, x, y)=\frac{f(x+y)}{\bar{F}(x)} f_{\delta}(0, x) .
$$

Equation (4.3) is a special case of a more general result, namely,

$$
h_{\delta}(u, x, y)=\frac{f(x+y)}{\bar{F}(x)} f_{\delta}(u, x)
$$

Equation (4.4) is interesting because it shows that the joint distribution of the surplus immediately prior to ruin and the deficit at ruin is determined by the individual claim amount distribution and the distribution of the surplus immediately prior to ruin. The intuition behind this is given in the proof of equation (2.40) of Gerber and Shiu (1998). We also note that the proof of Gerber and Shiu's equation still holds for equation (4.4). However, we will give an alternative analytical proof of equation (4.4) using equations for $h_{\delta}(u, x, y)$ and $f_{\delta}(u, x)$.

Due to equation (4.4), the study of the distribution of the surplus immediately prior to ruin is important. Dickson (1992) has found the following formulae for $f_{\delta=0}(u, x)$, which state that when $u \leq x$,

$$
f_{\delta=0}(u, x)=f_{\delta=0}(0, x) \frac{1-\psi(u)}{1-\psi(0)}
$$

and when $u>x$,

$$
f_{\delta=0}(u, x)=f_{\delta=0}(0, x) \frac{\psi(u-x)-\psi(u)}{1-\psi(0)} .
$$

Basically, formulae (4.5) and (4.6) show that the distribution of the surplus immediately prior to ruin is a function of the ruin probability $\psi$. Dickson (1992) also derived the corresponding formulae for $F_{\delta=0}(u, x)$. Gerber and Shiu (1998) have generalised Dickson's formulae under the definition of ruin probability given in their paper. Here we investigate whether Dickson's formulae hold when $\delta>0$. We will discuss this issue later in this section. 
First, differentiating equation (3.14) with respect to $x$ and $y$ successively, we get an integral equation for $h_{\delta}(u, x, y)$.

For any $u \geq 0$,

$$
h_{\delta}(u, x, y)=\frac{c h_{\delta}(0, x, y)}{c+\delta u}-\frac{\lambda I(u>x) f(x+y)}{c+\delta u}+\int_{0}^{u} k_{\delta}(u, t) h_{\delta}(t, x, y) d t .
$$

Letting $y \rightarrow \infty$ in equation (3.14), we get an integral equation for $F_{\delta}(u, x)$. For any $u \geq 0$,

$$
F_{\delta}(u, x)=\frac{c F_{\delta}(0, x)}{c+\delta u}-\frac{\lambda \mu}{c+\delta u} F_{1}(u \wedge x)+\int_{0}^{u} k_{\delta}(u, t) F_{\delta}(t, x) d t .
$$

Differentiating equation (4.8) with respect to $x$, we get an integral equation for $f_{\delta}(u, x)$. For any $u \geq 0$,

$$
f_{\delta}(u, x)=\frac{c f_{\delta}(0, x)}{c+\delta u}-\frac{\lambda I(u>x)}{c+\delta u} \bar{F}(x)+\int_{0}^{u} k_{\delta}(u, t) f_{\delta}(t, x) d t .
$$

Now let $K_{\delta}(x, s)$ be the resolvent of the Volterra equation (2.6), namely, for $x>s \geq 0$,

$$
K_{\delta}(x, s)=\sum_{m=1}^{\infty} k_{m, \delta}(x, s)
$$

where

$$
k_{m, \delta}(x, s)=\int_{s}^{x} k_{\delta}(x, t) k_{m-1, \delta}(t, s) d t, \quad m=2,3, \ldots
$$

with

$$
k_{1, \delta}(x, s)=k_{\delta}(x, s)=\frac{\delta+\lambda \bar{F}(x-s)}{c+\delta x} .
$$

Then by equations (3.12) and (2.8), we know that

$$
\bar{\psi}_{\delta}(u)=\frac{c \bar{\psi}_{\delta}(0)}{c+\delta u}+c \bar{\psi}_{\delta}(0) \int_{0}^{u} \frac{K_{\delta}(u, t)}{c+\delta t} d t
$$

implying that

$$
\int_{0}^{u} \frac{K_{\delta}(u, t)}{c+\delta t} d t=\frac{\bar{\psi}_{\delta}(u)}{c \bar{\psi}_{\delta}(0)}-\frac{1}{c+\delta u}
$$

In particular, when $\delta=0$

$$
k_{0}(x, s)=k_{\delta=0}(x, s)=\frac{\lambda}{c} \bar{F}(x-s) .
$$

Thus, for $x>s \geq 0$

$$
K_{0}(x, s)=K_{\delta=0}(x, s)=\sum_{n=1}^{\infty}(\lambda / c)^{n} \bar{F}^{* n}(x-s)=K_{0}(x-s) .
$$


In fact, $\theta K_{0}(u) /(1+\theta)$ is the density function of the compound geometric distribution function

$$
\sum_{n=0}^{\infty} \frac{\theta}{1+\theta}\left(\frac{1}{1+\theta}\right)^{n} F_{1}^{* n}(u)
$$

which is the well-known Beekman convolution formula for $\bar{\psi}(u)$.

Hence, equation (4.9) implies that for any $u \geq 0$,

$$
\int_{0}^{u} K_{0}(y) d y=\frac{\bar{\psi}(u)}{\bar{\psi}(0)}-1=\frac{\psi(0)-\psi(u)}{1-\psi(0)}
$$

Therefore, for any $u>x \geq 0$,

$$
\int_{0}^{x} K_{0}(u-t) d t=\int_{u-x}^{u} K_{0}(y) d y=\frac{\psi(u-x)-\psi(u)}{1-\psi(0)} .
$$

In addition, by equations $(3.11),(2.8)$, and (4.9), we know that $\psi_{\delta}(u)$ has the following representation of solution

$$
\begin{aligned}
\psi_{\delta}(u) & =\frac{c \psi_{\delta}(0)}{c+\delta u}-\frac{\lambda \mu}{c+\delta u} F_{1}(u)+\int_{0}^{u} K_{\delta}(u, t)\left(\frac{c \psi_{\delta}(0)}{c+\delta t}-\frac{\lambda \mu}{c+\delta t} F_{1}(t)\right) d t \\
& =\frac{c \psi_{\delta}(0)}{c+\delta u}-\frac{\lambda \mu}{c+\delta u} F_{1}(u)+c \psi_{\delta}(0)\left[\frac{\bar{\psi}_{\delta}(u)}{c \bar{\psi}_{\delta}(0)}-\frac{1}{c+\delta u}\right]-\lambda \mu \int_{0}^{u} \frac{K_{\delta}(u, t)}{c+\delta t} F_{1}(t) d t \\
& =\frac{\psi_{\delta}(0) \bar{\psi}_{\delta}(u)}{\bar{\psi}_{\delta}(0)}-\frac{\lambda \mu}{c+\delta u} F_{1}(u)-\lambda \mu \int_{0}^{u} \frac{K_{\delta}(u, t)}{c+\delta t} F_{1}(t) d t
\end{aligned}
$$

and hence

$$
\lambda \mu \int_{0}^{u} \frac{K_{\delta}(u, t)}{c+\delta t} F_{1}(t) d t=\frac{\psi_{\delta}(0) \bar{\psi}_{\delta}(u)}{\bar{\psi}_{\delta}(0)}-\psi_{\delta}(u)-\frac{\lambda \mu}{c+\delta u} F_{1}(u)
$$

We can now give equations satisfied by $F_{\delta}(u, x)$.

Theorem 4.1 When $u \leq x$,

$$
F_{\delta}(u, x)=\psi_{\delta}(u)-\frac{1-\psi_{\delta}(u)}{1-\psi_{\delta}(0)}\left(\psi_{\delta}(0)-F_{\delta}(0, x)\right)
$$

When $u>x$,

$$
F_{\delta}(u, x)=\left(F_{\delta}(0, x)-\frac{1}{1+\theta} F_{1}(x)\right) \frac{1-\psi_{\delta}(u)}{1-\psi_{\delta}(0)}+\lambda \mu \int_{0}^{x} \frac{K_{\delta}(u, t)}{c+\delta t}\left(F_{1}(x)-F_{1}(t)\right) d t .
$$


Proof. By equations (4.8), (2.8), and (4.9), we have

$$
\begin{aligned}
& F_{\delta}(u, x) \\
= & \frac{c F_{\delta}(0, x)}{c+\delta u}-\frac{\lambda \mu}{c+\delta u} F_{1}(u \wedge x)+\int_{0}^{u} K_{\delta}(u, t)\left[\frac{c F_{\delta}(0, x)}{c+\delta t}-\frac{\lambda \mu}{c+\delta t} F_{1}(t \wedge x)\right] d t \\
= & \frac{c F_{\delta}(0, x)}{c+\delta u}-\frac{\lambda \mu}{c+\delta u} F_{1}(u \wedge x)+c F_{\delta}(0, x) \int_{0}^{u} \frac{K_{\delta}(u, t)}{c+\delta t} d t \\
& -\lambda \mu \int_{0}^{u} \frac{K_{\delta}(u, t)}{c+\delta t} F_{1}(t \wedge x) d t \\
= & \frac{c F_{\delta}(0, x)}{c+\delta u}-\frac{\lambda \mu}{c+\delta u} F_{1}(u \wedge x)+c F_{\delta}(0, x)\left[\frac{\bar{\psi}_{\delta}(u)}{c \bar{\psi}_{\delta}(0)}-\frac{1}{c+\delta u}\right] \\
& -\lambda \mu \int_{0}^{u} \frac{K_{\delta}(u, t)}{c+\delta t} F_{1}(t \wedge x) d t \\
= & F_{\delta}(0, x) \frac{\bar{\psi}_{\delta}(u)}{\bar{\psi}_{\delta}(0)}-\frac{\lambda \mu}{c+\delta u} F_{1}(u \wedge x)-\lambda \mu \int_{0}^{u} \frac{K_{\delta}(u, t)}{c+\delta t} F_{1}(t \wedge x) d t .
\end{aligned}
$$

Thus, when $u \leq x$, by equations (4.14) and (4.11), we have,

$$
\begin{aligned}
F_{\delta}(u, x) & =F_{\delta}(0, x) \frac{\bar{\psi}_{\delta}(u)}{\bar{\psi}_{\delta}(0)}-\frac{\lambda \mu}{c+\delta u} F_{1}(u)-\lambda \mu \int_{0}^{u} \frac{K_{\delta}(u, t)}{c+\delta t} F_{1}(t) d t \\
& =F_{\delta}(0, x) \frac{\bar{\psi}_{\delta}(u)}{\bar{\psi}_{\delta}(0)}-\frac{\lambda \mu}{c+\delta u} F_{1}(u)-\left[\frac{\psi_{\delta}(0) \bar{\psi}_{\delta}(u)}{\bar{\psi}_{\delta}(0)}-\psi_{\delta}(u)-\frac{\lambda \mu}{c+\delta u} F_{1}(u)\right] \\
& =F_{\delta}(0, x) \frac{\bar{\psi}_{\delta}(u)}{\bar{\psi}_{\delta}(0)}-\frac{\psi_{\delta}(0) \bar{\psi}_{\delta}(u)}{\bar{\psi}_{\delta}(0)}+\psi_{\delta}(u) \\
& =\psi_{\delta}(u)-\frac{\bar{\psi}_{\delta}(u)}{\bar{\psi}_{\delta}(0)}\left(\psi_{\delta}(0)-F_{\delta}(0, x)\right),
\end{aligned}
$$

which implies that equation (4.12) holds.

On the other hand, when $u>x$, by equations (4.14) and (4.9), we have,

$$
\begin{aligned}
F_{\delta}(u, x)= & F_{\delta}(0, x) \frac{\bar{\psi}_{\delta}(u)}{\bar{\psi}_{\delta}(0)}-\frac{\lambda \mu}{c+\delta u} F_{1}(x)-\lambda \mu \int_{0}^{x} \frac{K_{\delta}(u, t)}{c+\delta t} F_{1}(t) d t-\lambda \mu \int_{x}^{u} \frac{K_{\delta}(u, t)}{c+\delta t} F_{1}(x) d t \\
= & F_{\delta}(0, x) \frac{\bar{\psi}_{\delta}(u)}{\bar{\psi}_{\delta}(0)}-\frac{\lambda \mu}{c+\delta u} F_{1}(x)-\lambda \mu \int_{0}^{x} \frac{K_{\delta}(u, t)}{c+\delta t} F_{1}(t) d t \\
& -F_{1}(x)\left[\lambda \mu \int_{0}^{u} \frac{K_{\delta}(u, t)}{c+\delta t} d t-\lambda \mu \int_{0}^{x} \frac{K_{\delta}(u, t)}{c+\delta t} d t\right] \\
= & F_{\delta}(0, x) \frac{\bar{\psi}_{\delta}(u)}{\bar{\psi}_{\delta}(0)}-\frac{\lambda \mu}{c+\delta u} F_{1}(x)-\lambda \mu F_{1}(x)\left[\frac{\bar{\psi}_{\delta}(u)}{c \bar{\psi}_{\delta}(0)}-\frac{1}{c+\delta u}\right] \\
& +\lambda \mu \int_{0}^{x} \frac{K_{\delta}(u, t)}{c+\delta t}\left(F_{1}(x)-F_{1}(t)\right) d t \\
= & \left(F_{\delta}(0, x)-\frac{1}{1+\theta} F_{1}(x)\right) \frac{1-\psi_{\delta}(u)}{1-\psi_{\delta}(0)}+\lambda \mu \int_{0}^{x} \frac{K_{\delta}(u, t)}{c+\delta t}\left(F_{1}(x)-F_{1}(t)\right) d t
\end{aligned}
$$


which gives equation (4.13).

Therefore, differentiating the equations for $F_{\delta}(u, x)$ in Theorem 4.1 with respect to $x$, we get the following generalisation of Dickson's formulae when $\delta>0$.

Theorem 4.2 When $u \leq x$,

$$
f_{\delta}(u, x)=\frac{1-\psi_{\delta}(u)}{1-\psi_{\delta}(0)} f_{\delta}(0, x)
$$

When $u>x$,

$$
f_{\delta}(u, x)=\left[f_{\delta}(0, x)-\frac{\lambda}{c} \bar{F}(x)\right] \frac{1-\psi_{\delta}(u)}{1-\psi_{\delta}(0)}+\lambda \bar{F}(x) \int_{0}^{x} \frac{K_{\delta}(u, t)}{c+\delta t} d t .
$$

Remark 4.1 When $\delta=0$, we have $f_{\delta}(0, x)=\frac{\lambda}{c} \bar{F}(x)$ and $K_{\delta}(u, t)=K_{0}(u-t)$. Thus, by equation (4.10), we find for $u>x$,

$$
\int_{0}^{x} \frac{K_{\delta}(u, t)}{c+\delta t} d t=\frac{1}{c} \int_{0}^{x} K_{0}(u-t) d t=\frac{\psi(u-x)-\psi(u)}{c(1-\psi(0))} .
$$

Therefore Theorem 4.2 generalises Dickson's formulae (4.5) and (4.6). However, we point out that for $u>x$, we cannot express $\int_{0}^{x}\left(K_{\delta}(u, t) /(c+\delta t)\right) d t$ in terms of $\psi_{\delta}(u)$. In this sense, Dickson's formula holds when $\delta>0$ only when $x \geq u$. Dickson interpreted his formulae using dual events, but the duality argument does not hold when $\delta>0$.

Next, we give an analytical proof of equation (4.4) based on the representation of solution for $h_{\delta}(u, x, y)$ and Theorem 4.2 .

Theorem 4.3 For any $u \geq 0$,

$$
h_{\delta}(u, x, y)=\frac{f(x+y)}{\tilde{F}(x)} f_{\delta}(u, x) .
$$

Proof. By equations (4.7), (2.8) and (4.9), we have

$$
\begin{aligned}
& h_{\delta}(u, x, y) \\
= & \frac{c h_{\delta}(0, x, y)}{c+\delta u}-\frac{\lambda I(u>x)}{c+\delta u} f(x+y)+\int_{0}^{u} K_{\delta}(u, t)\left[\frac{c h_{\delta}(0, x, y)}{c+\delta t}-\frac{\lambda I(t>x)}{c+\delta t} f(x+y)\right] d t \\
= & \frac{c h_{\delta}(0, x, y)}{c+\delta u}-\frac{\lambda I(u>x)}{c+\delta u} f(x+y)+c h_{\delta}(0, x, y)\left[\frac{\bar{\psi}_{\delta}(u)}{c \bar{\psi}_{\delta}(0)}-\frac{1}{c+\delta u}\right] \\
& -\lambda f(x+y) \int_{0}^{u} \frac{K_{\delta}(u, t)}{c+\delta t} I(t>x) d t \\
= & h_{\delta}(0, x, y) \frac{\bar{\psi}_{\delta}(u)}{\bar{\psi}_{\delta}(0)}-\frac{\lambda I(u>x)}{c+\delta u} f(x+y)-\lambda f(x+y) \int_{0}^{u} \frac{K_{\delta}(u, t)}{c+\delta t} I(t>x) d t .
\end{aligned}
$$


Thus, when $u \leq x$, by equations (4.16), (4.3), and Theorem 4.2, we have

$$
h_{\delta}(u, x, y)=h_{\delta}(0, x, y) \frac{\bar{\psi}_{\delta}(u)}{\bar{\psi}_{\delta}(0)}=f_{\delta}(0, x) \frac{f(x+y)}{\bar{F}(x)} \frac{\bar{\psi}_{\delta}(u)}{\bar{\psi}_{\delta}(0)}=f_{\delta}(u, x) \frac{f(x+y)}{\bar{F}(x)} .
$$

When $u>x$, by equations (4.16), (4.9), and (4.15), we have

$$
\begin{aligned}
h_{\delta}(u, x, y)= & h_{\delta}(0, x, y) \frac{\bar{\psi}_{\delta}(u)}{\bar{\psi}_{\delta}(0)}-\frac{\lambda}{c+\delta u} f(x+y)-\lambda f(x+y) \int_{x}^{u} \frac{K_{\delta}(u, t)}{c+\delta t} d t \\
= & h_{\delta}(0, x, y) \frac{\bar{\psi}_{\delta}(u)}{\bar{\psi}_{\delta}(0)}-\frac{\lambda}{c+\delta u} f(x+y)-\lambda f(x+y) \int_{0}^{u} \frac{K_{\delta}(u, t)}{c+\delta t} d t \\
& +\lambda f(x+y) \int_{0}^{x} \frac{K_{\delta}(u, t)}{c+\delta t} d t \\
= & h_{\delta}(0, x, y) \frac{\bar{\psi}_{\delta}(u)}{\bar{\psi}_{\delta}(0)}-\frac{\lambda}{c+\delta u} f(x+y)-\lambda f(x+y)\left[\frac{\bar{\psi}_{\delta}(u)}{c \bar{\psi}_{\delta}(0)}-\frac{1}{c+\delta u}\right] \\
& +\lambda f(x+y) \int_{0}^{x} \frac{K_{\delta}(u, t)}{c+\delta t} d t \\
= & {\left[h_{\delta}(0, x, y)-\frac{\lambda}{c} f(x+y)\right] \frac{\bar{\psi}_{\delta}(u)}{\bar{\psi}_{\delta}(0)}+\lambda f(x+y) \int_{0}^{x} \frac{K_{\delta}(u, t)}{c+\delta t} d t } \\
= & \left(h_{\delta}(0, x, y)-\frac{\lambda}{c} f(x+y)\right) \frac{1-\psi_{\delta}(u)}{1-\psi_{\delta}(0)} \\
& +\frac{f(x+y)}{\bar{F}(x)}\left\{f_{\delta}(u, x)-\left(f_{\delta}(0, x)-\frac{\lambda}{c} \bar{F}(x)\right) \frac{1-\psi_{\delta}(u)}{1-\psi_{\delta}(0)}\right\} \\
= & \frac{f(x+y)}{\bar{F}(x)} f_{\delta}(u, x)+\left(h_{\delta}(0, x, y)-\frac{f(x+y)}{\bar{F}(x)} f_{\delta}(0, x)\right) \frac{1-\psi_{\delta}(u)}{1-\psi_{\delta}(0)} \\
= & \frac{f(x+y)}{\bar{F}(x)} f_{\delta}(u, x)
\end{aligned}
$$

which completes the proof of Theorem 4.3.

In general, we can express $\Phi_{\delta}$ as a function of $\psi_{\delta}$ and $K_{\delta}$ as follows.

Theorem 4.4 For any $u \geq 0$,

$$
\Phi_{\delta}(u)=\left(\Phi_{\delta}(0)-\frac{\lambda}{c} \int_{0}^{u} A(s) d s\right) \frac{1-\psi_{\delta}(u)}{1-\psi_{\delta}(0)}+\lambda \int_{0}^{u} A(t) \int_{0}^{t} \frac{K_{\delta}(u, s)}{c+\delta s} d s d t .
$$

Proof. By equations (2.5) and (2.8), and (4.9) we have

$$
\begin{aligned}
& \Phi_{\delta}(u) \\
= & \frac{c \Phi_{\delta}(0)}{c+\delta u}-\frac{\lambda}{c+\delta u} \int_{0}^{u} A(s) d s+\int_{0}^{u} K_{\delta}(u, t)\left(\frac{c \Phi_{\delta}(0)}{c+\delta t}-\frac{\lambda}{c+\delta t} \int_{0}^{t} A(s) d s\right) d t
\end{aligned}
$$




$$
\begin{aligned}
= & \frac{c \Phi_{\delta}(0)}{c+\delta u}-\frac{\lambda}{c+\delta u} \int_{0}^{u} A(s) d s+c \Phi_{\delta}(0) \int_{0}^{u} \frac{K_{\delta}(u, t)}{c+\delta t} d t-\lambda \int_{0}^{u} \frac{K_{\delta}(u, t)}{c+\delta t} \int_{0}^{t} A(s) d s d t \\
= & \frac{c \Phi_{\delta}(0)}{c+\delta u}-\frac{\lambda}{c+\delta u} \int_{0}^{u} A(s) d s+c \Phi_{\delta}(0)\left(\frac{\bar{\psi}_{\delta}(u)}{c \bar{\psi}_{\delta}(0)}-\frac{1}{c+\delta u}\right)-\lambda \int_{0}^{u} \frac{K_{\delta}(u, t)}{c+\delta t} \int_{0}^{t} A(s) d s d t \\
= & \Phi_{\delta}(0) \frac{\bar{\psi}_{\delta}(u)}{\bar{\psi}_{\delta}(0)}-\frac{\lambda}{c+\delta u} \int_{0}^{u} A(s) d s-\lambda \int_{0}^{u} \frac{K_{\delta}(u, t)}{c+\delta t} \int_{0}^{t} A(s) d s d t \\
= & \Phi_{\delta}(0) \frac{\bar{\psi}_{\delta}(u)}{\bar{\psi}_{\delta}(0)}-\frac{\lambda}{c+\delta u} \int_{0}^{u} A(s) d s-\lambda \int_{0}^{u} \int_{0}^{t} A(s) d s d\left(\int_{0}^{t} \frac{K_{\delta}(u, s)}{c+\delta s} d s\right) \\
= & \Phi_{\delta}(0) \frac{\bar{\psi}_{\delta}(u)}{\bar{\psi}_{\delta}(0)}-\frac{\lambda}{c+\delta u} \int_{0}^{u} A(s) d s \\
& -\lambda\left(\int_{0}^{u} A(s) d s \int_{0}^{u} \frac{K_{\delta}(u, s)}{c+\delta s} d s-\int_{0}^{u} A(t) \int_{0}^{t} \frac{K_{\delta}(u, s)}{c+\delta s} d s d t\right) \\
= & \Phi_{\delta}(0) \frac{\bar{\psi}_{\delta}(u)}{\bar{\psi}_{\delta}(0)}-\frac{\lambda}{c+\delta u} \int_{0}^{u} A(s) d s \\
& -\lambda\left(\int_{0}^{u} A(s) d s\left(\frac{\bar{\psi}_{\delta}(u)}{c \bar{\psi}_{\delta}(0)}-\frac{1}{c+\delta u}\right)-\int_{0}^{u} A(t) \int_{0}^{t} \frac{K_{\delta}(u, s)}{c+\delta s} d s d t\right) \\
= & \left(\Phi_{\delta}(0)-\frac{\lambda}{c} \int_{0}^{u} A(s) d s\right) \frac{\bar{\psi}_{\delta}(u)}{\bar{\psi}_{\delta}(0)}+\lambda \int_{0}^{u} A(t) \int_{0}^{t} \frac{K_{\delta}(u, s)}{c+\delta s} d s d t
\end{aligned}
$$

which completes the proof of Theorem 4.4.

In particular, when $\delta=0$, we can obtain an expression for $\Phi=\Phi_{\delta=0}$, the expected value of the penalty function in the classical risk model, as a function of $\psi$ as follows.

Corollary 4.1 For any $u \geq 0$,

$$
\Phi(u)=\frac{\lambda}{c}\left(\int_{u}^{\infty} A(s) d s\right) \frac{1-\psi(u)}{1-\psi(0)}+\frac{\lambda}{c} \int_{0}^{u} A(t) \frac{\psi(u-t)-\psi(u)}{1-\psi(0)} d t
$$

Proof. By equations (4.17), (3.2), and (4.10), we have

$$
\begin{aligned}
\Phi(u) & =\left(\Phi(0)-\frac{\lambda}{c} \int_{0}^{u} A(s) d s\right) \frac{1-\psi(u)}{1-\psi(0)}+\frac{\lambda}{c} \int_{0}^{u} A(t) \int_{0}^{t} K_{0}(u-s) d s d t \\
& =\left(\frac{\lambda}{c} \int_{0}^{\infty} A(s) d s-\frac{\lambda}{c} \int_{0}^{u} A(s) d s\right) \frac{1-\psi(u)}{1-\psi(0)}-\frac{\lambda}{c} \int_{0}^{u} A(t) \frac{\psi(u-t)-\psi(u)}{1-\psi(0)} d t
\end{aligned}
$$

which gives equation (4.18).

Example 4.1 For $r \geq 0$, let $w\left(x_{1}, x_{2}\right)=e^{-r\left(x_{1}+x_{2}\right)}$. Then

$$
\Phi(u)=E\left(e^{-r\left(U\left(T^{-}\right)+|U(T)|\right)} I(T<\infty)\right)=\tilde{D}(u, r)
$$


the Laplace transform of the amount of the claim causing ruin when ruin occurs, where $T$ is the time of ruin when $\delta=0$. Then

$$
A(t)=\int_{t}^{\infty} w(t, x-t) d F(x)=\int_{t}^{\infty} e^{-r x} d F(x)
$$

and Corollary 4.1 gives

$$
\tilde{D}(u, r)=\frac{\lambda}{c}\left(\int_{u}^{\infty} \int_{t}^{\infty} e^{-r x} d F(x) d t\right) \frac{1-\psi(u)}{1-\psi(0)}+\frac{\lambda}{c} \int_{0}^{u}\left(\int_{t}^{\infty} e^{-r x} d F(x)\right) \frac{\psi(u-t)-\psi(u)}{1-\psi(0)} d t .
$$

Thus, given that ruin occurs, if the $(n+1)$-th moment of $F$ exists, we get the $n$-th moment of the claim causing ruin, namely,

$$
\begin{aligned}
& E\left(\left(U\left(T^{-}\right)+|U(T)|\right)^{n} \mid T<\infty\right)=\frac{(-1)^{n}}{\psi(u)}\left(\left.\frac{d^{n}}{d r^{n}} \tilde{D}(u, r)\right|_{r=0}\right) \\
= & \frac{\lambda}{c \psi(u)} \int_{u}^{\infty} \int_{t}^{\infty} x^{n} d F(x) d t \frac{1-\psi(u)}{1-\psi(0)}+\frac{\lambda}{c \psi(u)} \int_{0}^{u} \int_{t}^{\infty} x^{n} d F(x) \frac{\psi(u-t)-\psi(u)}{1-\psi(0)} d t .
\end{aligned}
$$

Corollary 4.1 is a very important result because we can use it to obtain many well known results from the literature. These include expressions for the ruin probability $\psi$, for the joint and marginal (defective) distributions of the surplus prior to ruin and the deficit at ruin, and for moments of these marginal distributions. For example, we can use Corollary 4.1 to obtain formula (4.5) of Lin and Willmot (2000) for the moments of the deficit at ruin, given that ruin occurs.

Finally, we can apply the methods of this section to the expected value of the discounted penalty function of Gerber and Shiu (1998). We will derive a formula similar to that in Corollary 4.1 in the next section.

\section{Gerber and Shiu's discounted penalty function re- visited}

Gerber and Shiu (1998) introduced the function

$$
\phi_{\alpha}(u)=\Phi_{\delta=0, \alpha}(u)=E\left(w\left(U\left(T^{-}\right),|U(T)|\right) e^{-\alpha T} I(T<\infty)\right),
$$

where $T$ is as defined in Example 4.1. Through this function we can study the joint distribution of surplus prior to ruin, the deficit at ruin and the time of ruin. They defined the following ruin function:

$$
\Psi_{\alpha}(u)=E\left(e^{-\alpha T+\rho U(T)} I(T<\infty)\right)
$$


where $\rho$ is the unique non-negative root of Lundberg's fundamental equation for the classical risk model, i.e.

$$
-\alpha+c \xi+\lambda\left(\int_{0}^{\infty} e^{-\xi x} f(x) d x-1\right)=0
$$

They showed that $\phi_{\alpha}(u)$ and $\Psi_{\alpha}(u)$ satisfy the following integral equations:

$$
\phi_{\alpha}(u)=h(u)+\int_{0}^{u} \phi_{\alpha}(x) g(u-x) d x
$$

and

$$
\Psi_{\alpha}(u)=\int_{u}^{\infty} e^{-\rho(x-u)} g(x) d x+\int_{0}^{u} \Psi_{\alpha}(x) g(u-x) d x
$$

where

$$
h(x)=\frac{\lambda}{c} \int_{x}^{\infty} e^{-\rho(t-x)} A(t) d t
$$

and

$$
g(x)=\frac{\lambda}{c} \int_{x}^{\infty} e^{-\rho(t-x)} f(t) d t .
$$

They also considered the following ruin function:

$$
\bar{\Psi}_{\alpha}(u)=e^{\rho u}-\Psi_{\alpha}(u) .
$$

It is easy to verify from equations (5.1) and (5.2) that $\bar{\Psi}_{\alpha}(u)$ satisfies the following integral equation:

$$
\bar{\Psi}_{\alpha}(u)=e^{\rho u} \bar{\Psi}_{\alpha}(0)+\int_{0}^{u} \bar{\Psi}_{\alpha}(x) g(u-x) d x
$$

where

$$
\bar{\Psi}_{\alpha}(0)=1-\Psi_{\alpha}(0)=1-\int_{0}^{\infty} e^{-\rho x} g(x) d x
$$

Thus, letting

$$
K_{\alpha}(x)=\sum_{n=1}^{\infty} g^{* n}(x)
$$

we have by equations (2.7) to (2.9)

$$
\bar{\Psi}_{\alpha}(u)=e^{\rho u} \bar{\Psi}_{\alpha}(0)+\bar{\Psi}_{\alpha}(0) \int_{0}^{u} e^{\rho x} K_{\alpha}(u-x) d x
$$


which is equation (4.12) of Gerber and Shiu (1997), an equation they obtained using Laplace transforms.

Equation (5.3) implies that

$$
\int_{0}^{u} K_{\alpha}(u-t) e^{\rho t} d t=\frac{\bar{\Psi}_{\alpha}(u)}{\bar{\Psi}_{\alpha}(0)}-e^{\rho u}
$$

or, equivalently,

$$
\int_{0}^{u} K_{\alpha}(y) e^{-\rho y} d y=\frac{e^{-\rho u} \bar{\Psi}_{\alpha}(u)-\bar{\Psi}_{\alpha}(0)}{\bar{\Psi}_{\alpha}(0)} .
$$

Thus, using arguments similar to those in the previous section, we can easily obtain an expression for $\phi_{\alpha}(u)$ as follows.

Theorem 5.1 For any $u \geq 0$,

$$
\phi_{\alpha}(u)=\frac{\lambda}{c} \int_{u}^{\infty} e^{-\rho t} A(t) d t \frac{e^{\rho u}-\Psi_{\alpha}(u)}{1-\Psi_{\alpha}(0)}+\frac{\lambda}{c} \int_{0}^{u} A(t) \frac{\Psi_{\alpha}(u-t)-e^{-\rho t} \Psi_{\alpha}(u)}{1-\Psi_{\alpha}(0)} d t .
$$

Proof. We have by equations (2.7) to (2.9)

$$
\begin{aligned}
\phi_{\alpha}(u)= & h(u)+\int_{0}^{u} K_{\alpha}(x) h(u-x) d x \\
= & h(u)+\int_{0}^{u} e^{\rho x} h(u-x) d\left(\int_{0}^{x} e^{-\rho t} K_{\alpha}(t) d t\right) \\
= & h(u)+h(0) e^{\rho u} \int_{0}^{u} e^{-\rho t} K_{\alpha}(t) d t-\frac{\lambda}{c} \int_{0}^{u} e^{\rho x} A(u-x) \int_{0}^{x} e^{-\rho t} K_{\alpha}(t) d t d x \\
= & h(u)+h(0) \frac{\bar{\Psi}_{\alpha}(u)-e^{\rho u} \bar{\Psi}_{\alpha}(0)}{\bar{\Psi}_{\alpha}(0)}-\frac{\lambda}{c} \int_{0}^{u} A(u-x) \frac{\bar{\Psi}_{\alpha}(x)-e^{\rho x} \bar{\Psi}_{\alpha}(0)}{\bar{\Psi}_{\alpha}(0)} d x \\
= & h(u)+h(0) \frac{e^{\rho u} \Psi_{\alpha}(0)-\Psi_{\alpha}(u)}{1-\Psi_{\alpha}(0)}-\frac{\lambda}{c} \int_{0}^{u} A(u-x) \frac{e^{\rho x} \Psi_{\alpha}(0)-\Psi_{\alpha}(x)}{1-\Psi_{\alpha}(0)} d x \\
= & \frac{\lambda}{c} \int_{u}^{\infty} e^{-\rho(t-u)} A(t) d t+\left(\frac{\lambda}{c} \int_{0}^{u} e^{-\rho t} A(t) d t+\frac{\lambda}{c} \int_{u}^{\infty} e^{-\rho t} A(t) d t\right) \frac{e^{\rho u} \Psi_{\alpha}(0)-\Psi_{\alpha}(u)}{1-\Psi_{\alpha}(0)} \\
& -\frac{\lambda}{c} \int_{0}^{u} A(t) \frac{e^{\rho(u-t)} \Psi_{\alpha}(0)-\Psi_{\alpha}(u-t)}{1-\Psi_{\alpha}(0)} d t \\
= & \frac{\lambda}{c} \int_{u}^{\infty} e^{-\rho t} A(t) d t \frac{e^{\rho u}-\Psi_{\alpha}(u)}{1-\Psi_{\alpha}(0)}+\frac{\lambda}{c} \int_{0}^{u} A(t) \frac{\Psi_{\alpha}(u-t)-e^{-\rho t} \Psi_{\alpha}(u)}{1-\Psi_{\alpha}(0)} d t .
\end{aligned}
$$

The above result gives an expression for $\phi_{\alpha}(u)$ as a function of the ruin probability $\Psi_{\alpha}(u)$ defined by Gerber and Shiu (1998). In fact, Corollary 4.1 is a special case of this result when $\alpha=0$. Also, Theorem 5.1 of Lin and Willmot (1999) can be obtained by taking $w\left(x_{1}, x_{2}\right)=I\left(x_{1} \leq x\right) I\left(x_{2} \leq y\right)$ in the above result. 


\section{Concluding remarks}

We have derived the following: an integral equation for $\Phi_{\delta, \alpha}(u)$; the Laplace transform of an auxiliary function of $\Phi_{\delta}(u)$; an exact expression for $\Phi_{\delta}(0)$; and relationships between ruin functions and the ultimate ruin probability. We have also generalised Dickson's (1992) formulae from the case when $\delta=0$ to the case when $\delta>0$. Ruin functions are very complicated when $\delta>0$. Although we have discussed some properties of ruin functions when $\delta>0$ we have been unable to find many explicit results. Further research in ruin theory when $\delta>0$ is clearly required. For example, it seems that we cannot apply both the probability measure transform technique of Gerber and Shiu (1998) and the Laplace transform technique of Sundt and Teugels (1995) to determine an expression for $\Phi_{\delta, \alpha}(0)$ when $\delta>0$ and $\alpha>0$. We leave this as an open question.

\section{References}

[1] Dickson, D. C. M. (1992) On the distribution of the surplus prior to ruin. Insurance: Math. Econom. 11, 191-207.

[2] Dufresne, F. and Gerber, H.U. (1988) The surpluses immediately before and at ruin, and the amount of the claim causing ruin. Insurance: Math. Econom. 7, 193-199.

[3] Gerber, H.U., Goovaerts, M.J. and Kaas, R. (1987) On the probability and severity of ruin. Astin Bulletin 17, 151-163.

[4] Gerber, H.U. and Shiu, E.S.W. (1997) The joint distribution of the time of ruin, the surplus immediately before ruin, and the deficit at ruin. Insurance: Math. Econom. 21, 129-137.

[5] Gerber, H.U. and Shiu, E.S.W. (1998) On the time value of ruin. North American Actuarial Journal 2: 48-78.

[6] Lin, X. and Willmot, G.E. (1999) Analysis of a defective renewal equation arising in ruin theory. Insurance: Math. Econom. 25, 63-84.

[7] Lin, X. and Willmot, G.E. (2000) The moments of the time of ruin, the surplus before ruin, and the deficit at ruin. Insurance: Math. Econom. 27, 19-44. 
[8] Mikhlin, S.G. (1957) Integral Equations. Pergamon Press, London.

[9] Sundt, B. and Teugels, J. L. (1995) Ruin estimates under interest force. Insurance: Math. Econom. 16, 7-22.

[10] Yang, H. and Zhang, L. (2001a) The joint distribution of surplus immediately before ruin and the deficit at ruin under interest force. Research Reports, No. 288, Department of Statistics and Actuarial Science, The University of Hong Kong.

[11] Yang, H. and Zhang, L. (2001b) On the distribution of the surplus immediately after ruin under interest force. Insurance: Math. Econom. 29, 247-256. 


\section{RESEARCH PAPER SERIES}

\begin{tabular}{|c|c|c|c|}
\hline No. & Date & Subject & Author \\
\hline 1 & MAR 1993 & $\begin{array}{l}\text { AUSTRALIAN SUPERANNUATION: } \\
\text { THE FACTS, THE FICTION, THE FUTURE }\end{array}$ & David M Knox \\
\hline 2 & APR 1993 & $\begin{array}{l}\text { AN EXPONENTIAL BOUND FOR RUIN } \\
\text { PROBABILITIES }\end{array}$ & David C M Dickson \\
\hline 3 & APR 1993 & $\begin{array}{l}\text { SOME COMMENTS ON THE COMPOUND } \\
\text { BINOMIAL MODEL }\end{array}$ & David C M Dickson \\
\hline 4 & AUG 1993 & RUIN PROBLEMS AND DUAL EVENTS & $\begin{array}{l}\text { David C M Dickson } \\
\text { Alfredo D Egídio dos } \\
\text { Reis }\end{array}$ \\
\hline 5 & SEP 1993 & $\begin{array}{l}\text { CONTEMPORARY ISSUES IN AUSTRALIAN } \\
\text { SUPERANNUATION - } \\
\text { A CONFERENCE SUMMARY }\end{array}$ & $\begin{array}{l}\text { David M Knox } \\
\text { John Piggott }\end{array}$ \\
\hline 6 & SEP 1993 & $\begin{array}{l}\text { AN ANALYSIS OF THE EQUITY INVESTMENTS } \\
\text { OF AUSTRALIAN SUPERANNUATION FUNDS }\end{array}$ & David M Knox \\
\hline 7 & OCT 1993 & $\begin{array}{l}\text { A CRITIQUE OF DEFINED CONTRIBUTION USING } \\
\text { A SIMULATION APPROACH }\end{array}$ & David M Knox \\
\hline 8 & JAN 1994 & REINSURANCE AND RUIN & $\begin{array}{l}\text { David C M Dickson } \\
\text { Howard R Waters }\end{array}$ \\
\hline 9 & MAR 1994 & $\begin{array}{l}\text { LIFETIME INSURANCE, TAXATION, EXPENDITURE } \\
\text { AND SUPERANNUATION (LITES): } \\
\text { A LIFE-CYCLE SIMULATION MODEL }\end{array}$ & $\begin{array}{l}\text { Margaret E Atkinson } \\
\text { John Creedy } \\
\text { David M Knox }\end{array}$ \\
\hline 10 & FEB 1994 & $\begin{array}{l}\text { SUPERANNUATION FUNDS AND THE } \\
\text { PROVISION OF DEVELOPMENT/VENTURE } \\
\text { CAPITAL: } \\
\text { THE PERFECT MATCH? YES OR NO }\end{array}$ & David M Knox \\
\hline 11 & JUNE 1994 & $\begin{array}{l}\text { RUIN PROBLEMS: SIMULATION OR } \\
\text { CALCULATION? }\end{array}$ & $\begin{array}{l}\text { David C M Dickson } \\
\text { Howard R Waters }\end{array}$ \\
\hline 12 & JUNE 1994 & $\begin{array}{l}\text { THE RELATIONSHIP BETWEEN THE AGE PENSION } \\
\text { AND SUPERANNUATION BENEFITS, } \\
\text { PARTICULARLY FOR WOMEN }\end{array}$ & David M Knox \\
\hline 13 & JUNE 1994 & $\begin{array}{l}\text { THE COST AND EQUITY IMPLICATIONS OF } \\
\text { THE INSTITUTE OF ACTUARIES OF AUSTRALIA } \\
\text { PROPOSED RETIREMENT INCOMES SRATEGY }\end{array}$ & $\begin{array}{l}\text { Margaret E Atkinson } \\
\text { John Creedy } \\
\text { David M Knox } \\
\text { Chris Haberecht }\end{array}$ \\
\hline 14 & SEPT 1994 & $\begin{array}{l}\text { PROBLEMS AND PROSPECTS FOR THE LIFE } \\
\text { INSURANCE AND PENSIONS SECTOR IN }\end{array}$ & $\begin{array}{l}\text { Catherine Prime } \\
\text { David M Knox }\end{array}$ \\
\hline
\end{tabular}


No.

16

17
OCT 1994

DEC 1994

Date

JAN 1995

FEB 1995

MAY 1995

JUNE 1995

JUNE 1995

JUNE 1995

SEP 1995

OCT 1995

DEC 1995

FEB 1996

FEB 1996

FEB 1996

MAR 1996
Subject

Author

PRESENT PROBLEMS AND PROSPECTIVE PRESSURES IN AUSTRALIA'S SUPERANNUATION SYSTEM

PLANNING RETIREMENT INCOME IN AUSTRALIA: ROUTES THROUGH THE MAZE

ON THE DISTRIBUTION OF THE DURATION OF NEGATIVE SURPLUS

OUTSTANDING CLAIM LIABILITIES: ARE THEY PREDICTABLE?

SOME STABLE ALGORITHMS IN RUIN THEORY AND THEIR APPLICATIONS

SOME FINANCIAL CONSEQUENCES OF THE SIZE OF AUSTRALIA'S SUPERANNUATION INDUSTRY IN THE NEXT THREE DECADES

MODELLING OPTIMAL RETIREMENT IN DECISIONS IN AUSTRALIA

AN EQUITY ANALYSIS OF SOME RADICAL SUGGESTIONS FOR AUSTRALIA'S RETIREMENT INCOME SYSTEM

EARLY RETIREMENT AND THE OPTIMAL RETIREMENT AGE

APPROXIMATE CALCULATIONS OF MOMENTS OF RUIN RELATED DISTRIBUTIONS

CONTEMPORARY ISSUES IN THE ONGOING REFORM OF THE AUSTRALIAN RETIREMENT INCOME SYSTEM

THE CHOICE OF EARLY RETIREMENT AGE AND THE AUSTRALIAN SUPERANNUATION SYSTEM

PREDICTIVE AGGREGATE CLAIMS DISTRIBUTIONS

THE AUSTRALIAN GOVERNMENT SUPERANNUATION CO-CONTRIBUTIONS: ANALYSIS AND COMPARISON

A SURVEY OF VALUATION ASSUMPTIONS AND FUNDING METHODS USED BY AUSTRALIAN ACTUARIES IN DEFINED BENEFIT SUPERANNUATION FUND VALUATIONS
David M Knox

Margaret E Atkinson John Creedy

David M Knox

David C M Dickson Alfredo D Egídio dos Reis

Ben Zehnwirth

David C M Dickson Alfredo D Egídio dos Reis

Howard R Waters

David M Knox

Margaret E Atkinson John Creedy

Margaret E Atkinson John Creedy

David M Knox

Angela Ryan

David C M Dickson

David M Knox

Margaret E Atkinson John Creedy

David C M Dickson Ben Zehnwirth

Margaret E Atkinson

Des Welch Shauna Ferris 


\begin{tabular}{|c|c|c|c|}
\hline No. & Date & Subject & Author \\
\hline 30 & MAR 1996 & $\begin{array}{l}\text { THE EFFECT OF INTEREST ON NEGATIVE } \\
\text { SURPLUS }\end{array}$ & $\begin{array}{l}\text { David C M Dickson } \\
\text { Alfredo D Egídio dos } \\
\text { Reis }\end{array}$ \\
\hline 31 & MAR 1996 & $\begin{array}{l}\text { RESERVING CONSECUTIVE LAYERS OF INWARDS } \\
\text { EXCESS-OFF-LOSS REINSURANCE }\end{array}$ & Greg Taylor \\
\hline 32 & AUG 1996 & $\begin{array}{l}\text { EFFECTIVE AND ETHICAL INSTITUTIONAL } \\
\text { INVESTMENT }\end{array}$ & Anthony Asher \\
\hline 33 & AUG 1996 & $\begin{array}{l}\text { STOCHASTIC INVESTMENT MODELS: UNIT } \\
\text { ROOTS, COINTEGRATION, STATE SPACE AND } \\
\text { GARCH MODELS FOR AUSTRALIA }\end{array}$ & $\begin{array}{l}\text { Michael Sherris } \\
\text { Leanna Tedesco } \\
\text { Ben Zehnwirth }\end{array}$ \\
\hline 34 & AUG 1996 & $\begin{array}{l}\text { THREE POWERFUL DIAGNOSTIC MODELS FOR } \\
\text { LOSS RESERVING }\end{array}$ & Ben Zehnwirth \\
\hline 35 & SEPT 1996 & $\begin{array}{l}\text { KALMAN FILTERS WITH APPLICATIONS TO LOSS } \\
\text { RESERVING }\end{array}$ & Ben Zehnwirth \\
\hline 36 & OCT 1996 & RELATIVE REINSURANCE RETENTION LEVELS & $\begin{array}{l}\text { David C M Dickson } \\
\text { Howard R Waters }\end{array}$ \\
\hline 37 & OCT 1996 & $\begin{array}{l}\text { SMOOTHNESS CRITERIA FOR MULTI- } \\
\text { DIMENSIONAL WHITTAKER GRADUATION }\end{array}$ & Greg Taylor \\
\hline 38 & OCT 1996 & $\begin{array}{l}\text { GEOGRAPHIC PREMIUM RATING BY WHITTAKER } \\
\text { SPATIAL SMOOTHING }\end{array}$ & Greg Taylor \\
\hline 39 & OCT 1996 & RISK, CAPITAL AND PROFIT IN INSURANCE & Greg Taylor \\
\hline 40 & OCT 1996 & $\begin{array}{l}\text { SETTING A BONUS-MALUS SCALE IN THE } \\
\text { PRESENCE OF OTHER RATING FACTORS }\end{array}$ & Greg Taylor \\
\hline 41 & NOV 1996 & $\begin{array}{l}\text { CALCULATIONS AND DIAGNOSTICS FOR LINK } \\
\text { RATION TECHNIQUES }\end{array}$ & $\begin{array}{l}\text { Ben Zehnwirth } \\
\text { Glen Barnett }\end{array}$ \\
\hline 42 & DEC 1996 & $\begin{array}{l}\text { VIDEO-CONFERENCING IN ACTUARIAL STUDIES - } \\
\text { A THREE YEAR CASE STUDY }\end{array}$ & David M Knox \\
\hline 43 & DEC 1996 & $\begin{array}{l}\text { ALTERNATIVE RETIREMENT INCOME } \\
\text { ARRANGEMENTS AND LIFETIME INCOME } \\
\text { INEQUALITY: LESSONS FROM AUSTRALIA }\end{array}$ & $\begin{array}{l}\text { Margaret E Atkinson } \\
\text { John Creedy } \\
\text { David M Knox }\end{array}$ \\
\hline 44 & JAN 1997 & $\begin{array}{l}\text { AN ANALYSIS OF PENSIONER MORTALITY BY } \\
\text { PRE-RETIREMENT INCOME }\end{array}$ & $\begin{array}{l}\text { David M Knox } \\
\text { Andrew Tomlin }\end{array}$ \\
\hline 45 & JUL 1997 & $\begin{array}{l}\text { TECHNICAL ASPECTS OF DOMESTIC LINES } \\
\text { PRICING }\end{array}$ & Greg Taylor \\
\hline 46 & AUG 1997 & $\begin{array}{l}\text { RUIN PROBABILITIES WITH COMPOUNDING } \\
\text { ASSETS }\end{array}$ & $\begin{array}{l}\text { David C M Dickson } \\
\text { Howard R Waters }\end{array}$ \\
\hline 47 & NOV 1997 & $\begin{array}{l}\text { ON NUMERICAL EVALUATION OF FINITE TIME } \\
\text { RUIN PROBABILITIES }\end{array}$ & David C M Dickson \\
\hline
\end{tabular}


JAN 1998

JAN 1998

MAR 1998

MAR 1998

MAR 1998

APR 1998

APR 1998

APR 1998

MAY 1998

MAY 1998

MAY 1998

JUNE 1998

JUNE 1998

JUNE 1998

JUNE 1998

AUG 1998

AUG 1998
ON THE MOMENTS OF RUIN AND RECOVERY TIMES

A DECOMPOSITION OF ACTUARIAL SURPLUS AND APPLICATIONS

PARTICIPATION PROFILES OF AUSTRALIAN WOMEN

PRICING THE STOCHASTIC VOLATILITY PUT OPTION OF BANKS' CREDIT LINE COMMITMENTS

ON ROBUST ESTIMATION IN BÜHLMANN STRAUB'S CREDIBILITY MODEL

AN ANALYSIS OF THE EQUITY IMPLICATIONS OF RECENT TAXATION CHANGES TO AUSTRALIAN SUPERANNUATION

TAX REFORM AND SUPERANNUATION - AN OPPORTUNITY TO BE GRASPED.

SUPER BENEFITS? ESTIMATES OF THE RETIREMENT INCOMES THAT AUSTRALIAN WOMEN WILL RECEIVE FROM SUPERANNUATION

A UNIFIED APPROACH TO THE STUDY OF TAIL PROBABILITIES OF COMPOUND DISTRIBUTIONS

THE DE PRIL TRANSFORM OF A COMPOUND $\mathbf{R}_{\mathbf{k}}$ DISTRIBUTION

ON MULTIVARIATE PANJER RECURSIONS

THE MULTIVARIATE DE PRIL TRANSFORM

ON ERROR BOUNDS FOR MULTIVARIATE DISTRIBUTIONS

THE EQUITY IMPLICATIONS OF CHANGING THE TAX BASIS FOR PENSION FUNDS

ACCELERATED SIMULATION FOR PRICING ASIAN OPTIONS

AN AFFINE PROPERTY OF THE RECIPROCAL ASIAN OPTION PROCESS

RUIN PROBLEMS FOR PHASE-TYPE(2) RISK PROCESSES

COMPARISON OF METHODS FOR EVALUATION OF THE $n$-FOLD CONVOLUTION OF AN ARITHMETIC DISTRIBUTION
Alfredo G Egídio dos Reis

Daniel Dufresne

M. E. Atkinson Roslyn Cornish

J.P. Chateau Daniel Dufresne

José Garrido Georgios Pitselis

David M Knox M. E. Atkinson Susan Donath

David M Knox

Susan Donath

Jun Cai José Garrido

Bjørn Sundt Okechukwu Ekuma

Bjørn Sundt

Bjørn Sundt

Bjørn Sundt

M E Atkinson

John Creedy

David Knox

Felisa J Vázquez-Abad Daniel Dufresne

Daniel Dufresne

David C M Dickson Christian Hipp

Bjørn Sundt David C M Dickson 


\begin{tabular}{|c|c|c|c|}
\hline No. & Date & Subject & Author \\
\hline 66 & NOV 1998 & $\begin{array}{l}\text { COMPARISON OF METHODS FOR EVALUATION OF } \\
\text { THE CONVOLUTION OF TWO COMPOUND } R_{1} \\
\text { DISTRIBUTIONS }\end{array}$ & $\begin{array}{l}\text { David C M Dickson } \\
\text { Bjørn Sundt }\end{array}$ \\
\hline 67 & NOV 1998 & $\begin{array}{l}\text { PENSION FUNDING WITH MOVING AVERAGE } \\
\text { RATES OF RETURN }\end{array}$ & $\begin{array}{l}\text { Diane Bédard } \\
\text { Daniel Dufresne }\end{array}$ \\
\hline 68 & DEC 1998 & $\begin{array}{l}\text { MULTI-PERIOD AGGREGATE LOSS } \\
\text { DISTRIBUTIONS FOR A LIFE PORTFOLIO }\end{array}$ & $\begin{array}{l}\text { David C M Dickson } \\
\text { Howard R Waters }\end{array}$ \\
\hline 69 & FEB 1999 & $\begin{array}{l}\text { LAGUERRE SERIES FOR ASIAN AND OTHER } \\
\text { OPTIONS }\end{array}$ & Daniel Dufresne \\
\hline 70 & MAR 1999 & $\begin{array}{l}\text { THE DEVELOPMENT OF SOME CHARACTERISTICS } \\
\text { FOR EQUITABLE NATIONAL RETIREMENT } \\
\text { INCOME SYSTEMS }\end{array}$ & $\begin{array}{l}\text { David Knox } \\
\text { Roslyn Cornish }\end{array}$ \\
\hline 71 & APR 1999 & $\begin{array}{l}\text { A PROPOSAL FOR INTEGRATING AUSTRALIA'S } \\
\text { RETIREMENT INCOME POLICY }\end{array}$ & David Knox \\
\hline 72 & NOV 1999 & $\begin{array}{l}\text { THE STATISTICAL DISTRIBUTION OF INCURRED } \\
\text { LOSSES AND ITS EVOLUTION OVER TIME I: NON- } \\
\text { PARAMETRIC MODELS }\end{array}$ & Greg Taylor \\
\hline 73 & NOV 1999 & $\begin{array}{l}\text { THE STATISTICAL DISTRIBUTION OF INCURRED } \\
\text { LOSSES AND ITS EVOLUTION OVER TIME II: } \\
\text { PARAMETRIC MODELS }\end{array}$ & Greg Taylor \\
\hline 74 & DEC 1999 & $\begin{array}{l}\text { ON THE VANDERMONDE MATRIX AND ITS ROLE } \\
\text { IN MATHEMATICAL FINANCE }\end{array}$ & Ragnar Norberg \\
\hline 75 & DEC 1999 & A MARKOV CHAIN FINANCIAL MARKET & Ragnar Norberg \\
\hline 76 & MAR 2000 & $\begin{array}{l}\text { STOCHASTIC PROCESSES: LEARNING THE } \\
\text { LANGUAGE }\end{array}$ & $\begin{array}{l}\text { A J G Cairns } \\
\text { D C M Dickson } \\
\text { A S Macdonald } \\
\text { H R Waters } \\
\text { M Willder }\end{array}$ \\
\hline 77 & MAR 2000 & $\begin{array}{l}\text { ON THE TIME TO RUIN FOR ERLANG(2) RISK } \\
\text { PROCESSES }\end{array}$ & David C M Dickson \\
\hline 78 & JULY 2000 & RISK AND DISCOUNTED LOSS RESERVES & Greg Taylor \\
\hline 79 & JULY 2000 & STOCHASTIC CONTROL OF FUNDING SYSTEMS & Greg Taylor \\
\hline 80 & NOV 2000 & $\begin{array}{l}\text { MEASURING THE EFFECTS OF REINSURANCE BY } \\
\text { THE ADJUSTMENT COEFFICIENT IN THE SPARRE } \\
\text { ANDERSON MODEL }\end{array}$ & $\begin{array}{l}\text { Maria de Lourdes } \\
\text { Centeno }\end{array}$ \\
\hline 81 & NOV 2000 & $\begin{array}{l}\text { THE STATISTICAL DISTRIBUTION OF INCURRED } \\
\text { LOSSES AND ITS EVOLUTION OVER TIME } \\
\text { III: DYNAMIC MODELS }\end{array}$ & Greg Taylor \\
\hline
\end{tabular}


No.

Date

82

DEC 2000

83

84

85

86

87

88

89

91
NOVEMBER 2001

NOVEMBER 2001

DEC 2000

FEB 2001

FEB 2001

JUNE 2001

SEPTEMBER 2001

NOVEMBER 2001

NOVEMBER 2001
Subject

Author

OPTIMAL INVESTMENT FOR INVESTORS WITH STATE DEPENDENT INCOME, AND FOR INSURERS

HEDGING IN INCOMPLETE MARKETS AND OPTIMAL CONTROL

DISCRETE TIME RISK MODELS UNDER STOCHASTIC FORCES OF INTEREST

MODERN LANDMARKS IN ACTUARIAL SCIENCE Inaugural Professorial Address

LUNDBERG INEQUALITIES FOR RENEWAL EQUATIONS

VOLATILITY, BETA AND RETURN

WAS THERE EVER A MEANINGFUL

RELATIONSHIP?

EXPLICIT, FINITE TIME RUIN PROBABILITIES FOR DISCRETE, DEPENDENT CLAIMS

ON THE DISTRIBUTION OF THE DEFICIT AT RUIN WHEN CLAIMS ARE PHASE-TYPE

THE INTEGRATED SQUARE-ROOT PROCESS

ON THE EXPECTED DISCOUNTED PENALTY FUNCTION AT RUIN OF A SURPLUS PROCESS WITH INTEREST
Christian Hipp

Christian Hipp

Michael Taksar

Jun Cai

David C M Dickson

Gordon E Willmot

Jnun Cai

$\mathrm{X}$ Sheldon Lin

Richard Fitzherbert

Zvetan G Ignatov Vladimir K Kaishev Rossen S Krachunov

Steve Drekic David C M Dickson David A Stanford Gordon E Willmot

Daniel Dufresne

Jun Cai

David C M Dickson 


\section{University Library}

\section{- M M I E R R A A gateway to Melbourne's research publications}

Minerva Access is the Institutional Repository of The University of Melbourne

Author/s:

Cai, Jun;Dickson, David C.M.

Title:

On the expected discounted penalty function at ruin of a surplus process with interest

Date:

2001-11

Citation:

Cai, Jun and Dickson, David C.M. (2001) On the expected discounted penalty function at ruin of a surplus process with interest.

Persistent Link:

http://hdl.handle.net/11343/33692 